\title{
ARTICLE
}

Received 4 Sep 2012 | Accepted 10 Apr 2013 | Published 21 May $2013 \quad$ DOI: 10.1038/ncomms2869

\section{Gravity-induced encapsulation of liquids by destabilization of granular rafts}

Manouk Abkarian ${ }^{1, \star}$, Suzie Protière ${ }^{2, \star}$, Jeffrey M. Aristoff ${ }^{3, \dagger} \&$ Howard A. Stone ${ }^{3}$

Droplets and bubbles coated by a protective armour of particles find numerous applications in encapsulation, stabilization of emulsions and foams, and flotation techniques. Here we study the role of a body force, such as in flotation, as a means of continuous encapsulation by particles. We use dense particles, which self-assemble into rafts, at oil-water interfaces. We show that these rafts can be spontaneously or controllably destabilized into armoured oil-inwater droplets, which highlights a possible role for common granular materials in environmental remediation. We further present a method for continuous production and discuss the generalization of our approach towards colloidal scales.

\footnotetext{
${ }^{1}$ Université Montpellier 2-CNRS, Laboratoire Charles Coulomb, UMR 5221, Cedex 5, Montpellier F-34095, France. ${ }^{2}$ CNRS, Université Paris 06, Institut Jean le Rond d'Alembert, UMR 7190, Paris F-75005, France. ${ }^{3}$ Department of Mechanical and Aerospace Engineering, Princeton University, Princeton, New Jersey 08544, USA. * These authors contributed equally to this work. †Present address: Numerica Corporation, 4850 Hahns Peak Drive, Suite 200 , Loveland, Colorado 80538, USA. Correspondence and requests for materials should be addressed to M.A. (email: manouk.abkarian@univ-montp2.fr) or to H.A.S. (email: hastone@princeton.edu).
} 
nterfaces can have two roles in organizing the assembly of particles. Either they serve as a template on which particles aggregate due to direct mutual interactions or the interfaces themselves mediate the interaction by capillary forces due to local deformations. Such local surface deformations arise, for instance, when the contact line around a particle is not planar due to shape anisotropy (for example, rods, ellipsoids and hexagons ${ }^{1-4}$ ) or surface roughness 5,6 . Other methods for assembly use external forces that can induce local interface deformation by acting on the particles using body forces such as gravity ${ }^{7}$, electric $^{8}$ or magnetic fields ${ }^{9}$ (Fig. 1a,b). Such capillary-driven aggregation $^{10,11}$, as well as the properties of particle-laden interfaces $^{12}$, has been studied extensively, but always in a parameter range limited to small body forces, which produce stable particle aggregates or rafts.

However, in the case of large flotation forces, the interface may undergo strong deformations and the particle rafts may become unstable. The stability of similar objects has only been studied in one dimension ${ }^{13}$. Here we document how the instability of twodimensional configurations leads to the encapsulation of one fluid phase into transportable droplets protected by a heavy granular armour (see schematic in Fig. 1c). The resulting armoured droplets are distinct from liquid marbles ${ }^{14}$ and colloidal armoured droplets ${ }^{15}$ where the weight of the coating material is negligible. At the colloidal scale, methods for producing particlecovered interfaces rely on emulsification where particles are trapped by rapidly moving interfaces during phase separation ${ }^{16}$ or droplet formation ${ }^{17,18}$. Above the colloidal scale, formation methods remain scarce and mainly consist of rolling droplets on beds of grains ${ }^{14,19,20}$. Our new method allows for a rapid and continuous production of droplets. Finally, we show how to control the production of armoured droplets so that the size of the particles used, the amount of liquid encapsulated and the size of the armour can be varied independently. Our results suggest a simple approach in which common granular materials may be used for encapsulation ${ }^{21,22}$, handling and containment of hazardous or volatile liquids. It may also help improve the stabilization of emulsions ${ }^{23}$ and foams ${ }^{1}$, as well as flotation techniques ${ }^{24}$. Other applications include the surface catalysis of chemical reactions, as well as the remediation of hazardous materials such as an oil spill.

\section{Results}

The destabilization of a granular raft. In a typical experiment we deposit a layer of oil (density $\rho_{\mathrm{o}}$ ) on a bath of water (density $\rho_{\mathrm{w}}$ ) and add heavy particles (Fig. 1a-d and Methods). We observe two different behaviours depending on the manner of particle addition. In the first case, we sprinkle ordinary dry sand or other spherical particles, with density $\rho_{\mathrm{s}}$ and radius $a$, which are typically a few hundred microns or larger, into the oil. The particles cross the oil-air interface and sediment through the oil, until they come to rest at the oil-water interface (surface tension $\gamma_{\text {ow }}$ ) because of capillary and buoyancy forces. The particles experience long-range capillary attractions ${ }^{25}$, which lead to the formation of a close-packed particle layer, which we call a capillary raft; a stable raft is shown in Fig. 1e. Simultaneously, the dense particle layer bends the interface downwards on a distance close to but smaller than the capillary length $\ell_{\mathrm{c}}=\sqrt{\gamma_{\mathrm{ow}} /\left(\rho_{\mathrm{w}}-\rho_{\mathrm{o}}\right) g}$ $\approx 5.6 \mathrm{~mm}$, where $g$ is the gravitational acceleration.

For a given particle density and size, when the number of particles in the raft is large enough, the raft may become unstable and sink, encapsulating the lower-density oil phase within a nearly close-packed particle-laden interface that is surrounded by water (Fig. 1f,g). The raft destabilization depends mainly on the balance between the local buoyancy forces and the capillary forces
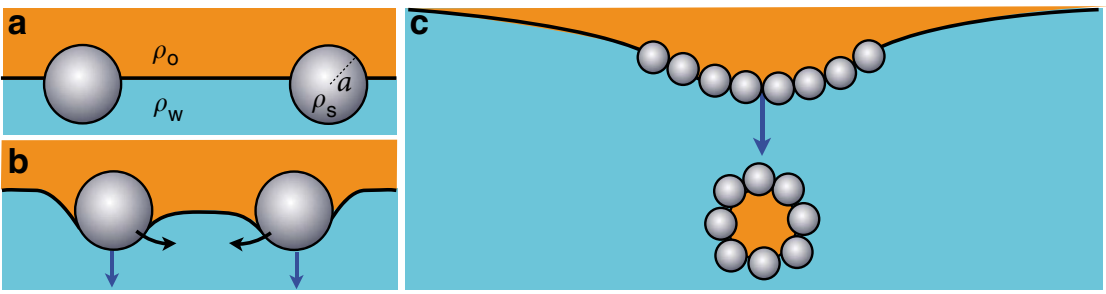

d

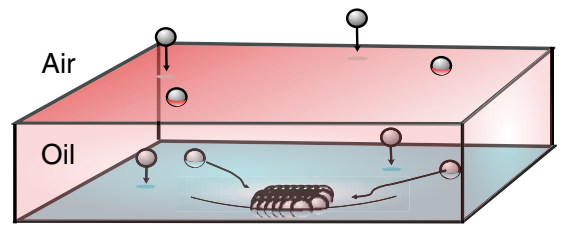

Water
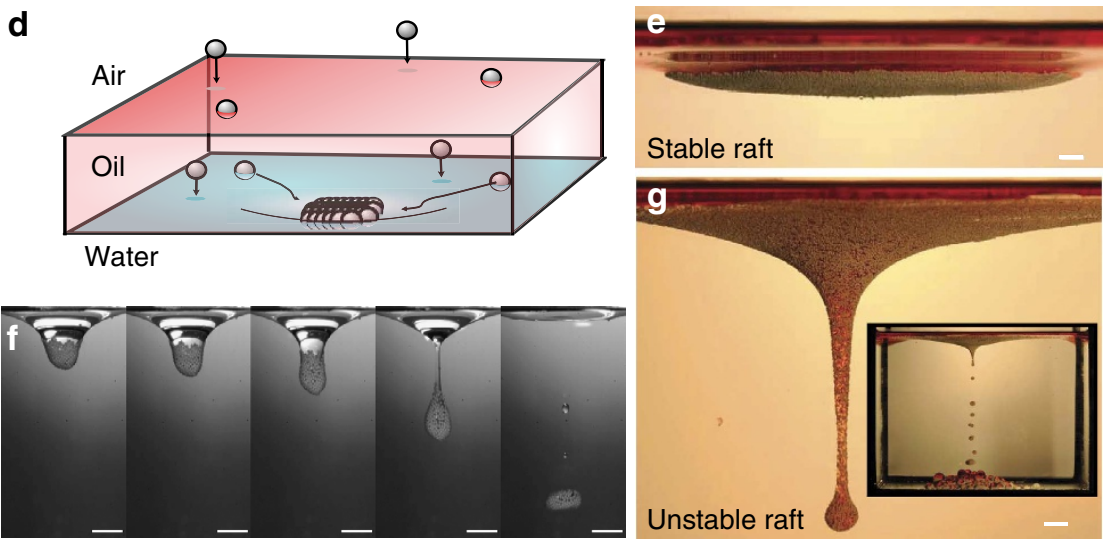

Figure 1 | Formation of armoured droplets from a raft of particles. Particles at an oil/water interface (a) interact via capillarity (b) to form a raft that can destabilize (c). (d) Schematic of the experimental setup: a container is filled with water on top of which a layer of mineral oil (red) is deposited $\left(\gamma_{o w}=51 \mathrm{mN} \mathrm{m}^{-1}\right)$. Spherical particles are sprinkled uniformly from above and collect in a near close-packed arrangement at the oil-water interface, owing to capillary and buoyancy forces. Depending on the number of particles, the capillary raft either (e) remains floating at the oil-water interface or

$(\mathbf{f}, \mathbf{g})$ destabilizes. There are two possible destabilization scenarios: the raft (f) encapsulates a single drop of oil and sinks entirely or ( $\mathbf{g}$ ) forms a long jet, which breaks into a series of armoured droplets, thereby transporting oil in the form of encapsulated drops to the bottom of the container. The experiments use dense particles. For $\mathbf{f}$, density $\rho_{\mathrm{s}}=6,000 \mathrm{~kg} \mathrm{~m}^{-3}$, radius $a=0.2 \mathrm{~mm}$ and time lapse between pictures $0.05 \mathrm{~s}$. Scale bar, $5 \mathrm{~mm}$. For $\mathbf{e}$ and $\mathbf{g}$, density $\rho_{\mathrm{s}}=3,800 \mathrm{~kg} \mathrm{~m}^{-3}$, radius $a=0.175 \pm 0.025 \mathrm{~mm}$. Scale bar, $10 \mathrm{~mm}$. 
at the border of the raft. We observe that depending on the size and density of the particles, the raft can either extend horizontally on the scale of $\ell_{\mathrm{c}}$ or for smaller or less dense particles the horizontal scale of deformation can extend several times the capillary length. These possibilities lead to two types of destabilization: (i) a single covered droplet (Fig. 1f, Supplementary Movie 1) or (ii) an 'interfacial granular jet' that breaks due to a Rayleigh-Plateau-like instability (Fig. 1g and Supplementary Movie 2), which results in the continuous formation of millimetre-sized 'armoured' oil droplets. Typically, the jet continues sinking until almost the entire particle raft has disappeared and carries along with it a much larger volume of the oil. Furthermore, although the encapsulated oil is surrounded by the denser water, the particle-covered droplets remain indefinitely at the bottom of the tank, owing to the weight of the particles. We have also observed that motion of the water phase due to stirring or surface waves (amplitude much larger than the thickness of the oil film or the particle size) does not release the oil phase from the droplets when their surface is fully covered and the drop size is smaller than $\ell_{\mathrm{c}}$.

Stability and shape of armoured droplets. We conducted experiments to probe the shape and stability of these heavy armoured droplets. In addition, we developed a detailed mathematical model, as sketched in Fig. 2, to rationalize the quantitative trends as material and geometric parameters are varied (the basic equations are discussed in Methods). Briefly, we model the armoured droplet as an axisymmetric continuum body that can support tension with consideration of both particle-covered and particle-free portions of the interface. The formulation introduces the surface area $\Sigma$ of this continuous shell and the volume of encapsulated fluid $V_{\text {oil }}=\frac{4}{3} \pi R_{0}^{3}$, which serves to introduce the effective drop radius $R_{0}$. We let the origin of the coordinate system be the apex of the droplet and denote the interface by $h(r)$, where $h$ is the depth and $r$ is the radial coordinate (see Fig. 2). Along the liquid-liquid interface, the tension is constant and equal to the interfacial tension $\gamma_{\text {ow }}$, whereas along the particle-laden portion of the interface, the effective tension varies due to the mass of the particle shell. The equilibrium shape of the

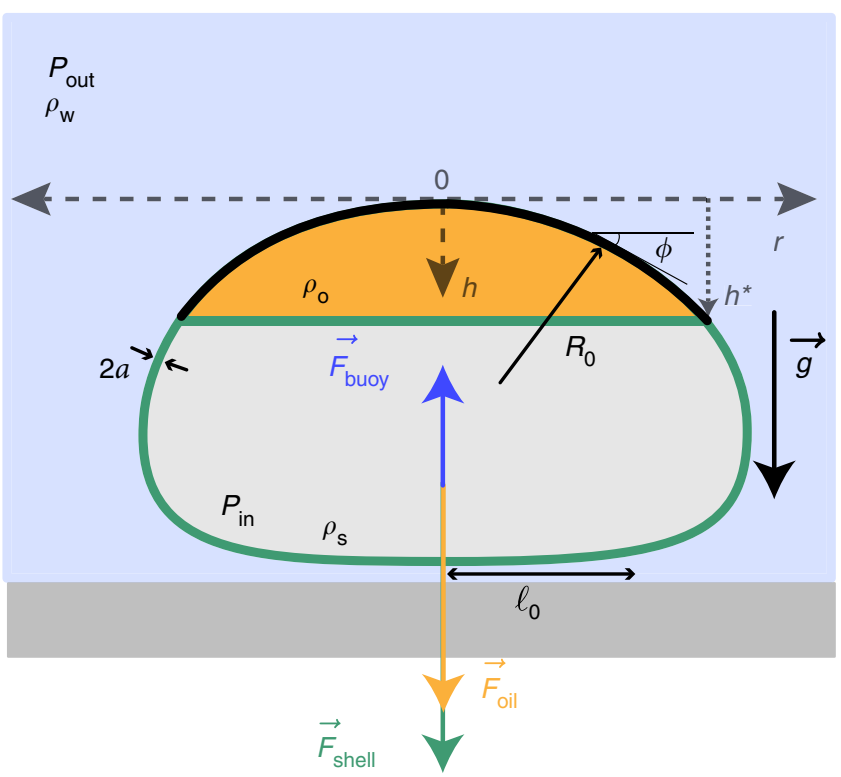

Figure 2 | Notation used for the mathematical model of the shape of an oil droplet contained by a shell of particles and surrounded by water. The model is developed based on a normal stress balance (Young-Laplace equation) at the liquid-liquid interface, and normal and tangential stress balances at the particle-laden interface. interface is determined by writing equations for the normal and tangential stress balances along the particle-laden interface and the normal stress balance at the liquid-liquid interface (see Methods). These steps yield a nonlinear boundary-value problem, which we solve numerically. From these equations, we identify the dimensionless parameter

$$
\mathcal{D}=\left(\frac{\rho_{\mathrm{s}}-\rho_{\mathrm{w}}}{\rho_{\mathrm{w}}-\rho_{\mathrm{o}}}\right) \frac{b_{\text {eff }}}{\ell_{\mathrm{c}}}
$$

where $b_{\text {eff }}$ is the weight-averaged effective thickness of the armour; $b_{\text {eff }}=(4 / 3) a \eta$ where close packing of the particles corresponds to $\eta=0.85$.

With this approach, we can predict the shape of an armoured drop (Fig. 3a), and, more significantly, we have a tool for identifying optimum conditions for encapsulation. We compare experiments and theory for the shape evolution of an armoured drop, where in the experiments we add oil to a droplet using a syringe (photographs in Fig. 3a). As the oil volume is increased, the droplet height increases monotonically, whereas the droplet width $w$ first increases before reaching a plateau (Fig. 3a). Both trends are reproduced quantitatively by our numerical model. Qualitatively, the height of the droplet increases with droplet volume as a consequence of buoyancy, and both experimental measurements of height and width show some slight deviation from the model at the largest volumes, which we believe may be a consequence of compression effects between the particles that are neglected in the model.

As we increase the oil volume in the shell, $V_{\text {oil }}$, and thus the buoyancy force, the contact area, $\pi \ell_{0}^{2}$, between the droplet and the bottom of the tank decreases (Fig. 3b). This observation may be understood by a reduced model in which the drop is assumed to be spherical (radius $R_{0}$ ), and by prescribing a vertical force balance over the contact region. The drop gains energy by lowering its centre of gravity and so develops a surface of contact between the drop and the solid on which it is placed. The solid experiences a pressure difference $\Delta P$ corresponding to its reaction to the contact disc to balance the weight of the drop (the weight of the oil and its shell), as well as the buoyancy force that acts against it (Fig. 2). Assuming that the particles are mostly in the water phase, this gives:

$$
\Delta P \pi \ell_{0}^{2}=F_{\text {oil }}-F_{\text {buoy }}+F_{\text {shell }}
$$

We have $\quad F_{\text {oil }}-F_{\text {buoy }}=\frac{4}{3} \pi R_{0}^{3}\left(\rho_{\mathrm{o}}-\rho_{\mathrm{w}}\right) g \quad$ and $\quad F_{\text {shell }}=\Omega_{\mathrm{s}}$ $\left(\rho_{\mathrm{s}}-\rho_{\mathrm{w}}\right) g$ with $\Omega_{\mathrm{s}}$, the volume of the drop's shell, that is, $\Omega_{\mathrm{s}}=b_{\text {eff }} \Sigma$. According to the Young-Laplace equation: $\Delta P=\left(2 \gamma_{\text {ow }} / R_{0}\right)$. Substituting these results in equation (2) yields an expression for the contact length: $\ell_{0} / \ell_{c}=\sqrt{(2 / 3)}$ Bo $\sqrt{\left(3 \Sigma / 4 \pi \ell_{\mathrm{c}}^{2}\right)\left(\mathcal{D} / \mathrm{Bo}^{3 / 2}\right)-1}$, where $\mathrm{Bo}=\left(R_{0} / \ell_{\mathrm{c}}\right)^{2}$ is the Bond number. Because the drop is not a perfect sphere, our reduced model overestimates the volume of oil for a given Bond number. To compensate this effect, we overestimate the weight of the shell by taking $b_{\text {eff }}=2 a$, which leads to a good agreement between the reduced model and experiment, as shown in Fig. 3b. As the drop elongates vertically and reaches the limit of containment of the shell, we observe two scenarios for instability at a critical value of the Bond number: either the entire droplet becomes buoyant and floats to the surface (blue curve, Fig. $3 \mathrm{~b}$ and complete shape evolution in Supplementary Fig. S1a) or the uncovered portion of the droplet pinches off and rises to the surface, leaving behind a smaller armoured droplet (red curve, Fig. 3b and complete shape evolution in Supplementary Fig. S1b). Flotation occurs if the buoyancy force exceeds the weight of the particles, that is, when $\ell_{0} / \ell_{c}=0$, leading to the condition $3 \Sigma \mathcal{D} / 4 \pi \ell_{\mathrm{c}}^{2} \leq \mathrm{Bo}^{3 / 2}$.

In contrast, when the surface area covered by the particles is increased, the buoyancy force on the droplet exceeds the surface 


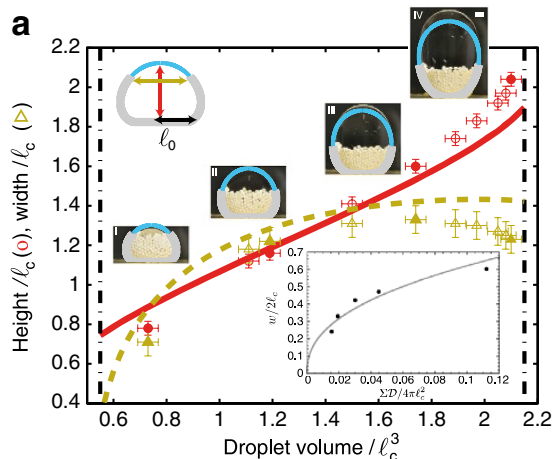

b

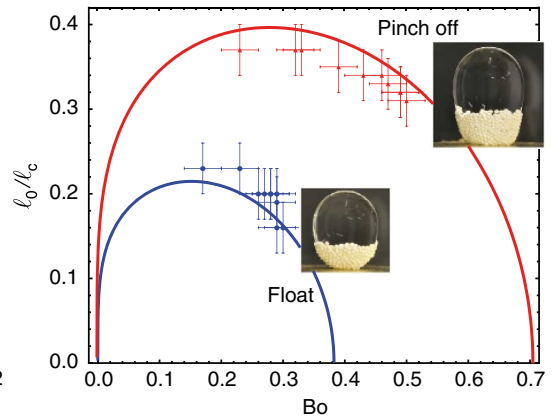

C

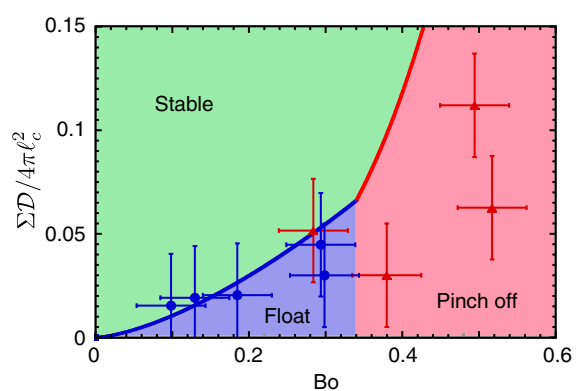

Figure 3 | From complete to partial encapsulation and the boundaries of stability. (a) Mineral oil is progressively added to a single-armoured droplet produced by the destabilization of a capillary raft. Four photographs of the armoured drop are shown, along with the numerically calculated shapes. The droplet is covered by approximately 700 zirconia particles (this gives $\Sigma=0.75 \mathrm{~cm}^{2}$ for particles of radius $a=0.175 \pm 0.025 \mathrm{~mm}, \rho_{\mathrm{s}}=3,800 \mathrm{~kg} \mathrm{~m}-3$ and $\ell_{c}=5.6 \mathrm{~mm}$ ). Scale bar, $1 \mathrm{~mm}$. As mineral oil is added to the drop, the height of the drop increases monotonically (circles), whereas the width first increases and then decreases (triangles). The filled symbols correspond to images (a). The theory captures the trends for the height (solid curve) and width (dashed curve) and characterizes the range of droplet volumes over which encapsulation of the upper layer fluid is possible $(0.077-0.28 \mathrm{ml}$ or $0.6-2.2$ in

dimensionless units). The inset corresponds to the measure of the critical width $w$ just before floating or pinching off as a function of $\Sigma \mathcal{D} / 4 \pi \ell_{c}^{2}$ with $\mathcal{D}=\left(\rho_{\mathrm{s}}-\rho_{\mathrm{w}} / \rho_{\mathrm{w}}-\rho_{\mathrm{o}}\right) 2 a / \ell_{\mathrm{c}}$ and $\Sigma$, the surface area of the armoured region of a droplet. The line is a fit with the power law: $\left(w / 2 \ell_{\mathrm{c}}\right)=$ $1.62\left(\Sigma \mathcal{D} / 4 \pi \ell_{c}^{2}\right)^{0.42}$. (b) The contact line $\ell_{0}$ first increases with the Bond number Bo and then decreases as mineral oil is added to the droplet. The solid lines correspond to the behaviour predicted by the model (see equation (2)). The two curves show the evolution of the contact line $\ell_{0} / \ell_{c}$ as a function of Bo for armoured droplets of different $\Sigma$ (blue curve: $\Sigma=0.30 \mathrm{~cm}^{2}$, red curve $\Sigma=0.75 \mathrm{~cm}^{2}$ ). When $V_{\text {oil }}$ is increased, the drop with the smaller $\Sigma$ becomes buoyant and floats back to the surface (blue curve), whereas for the other drop, the uncovered part pinches off and rises to the surface leaving behind a smaller completely covered armoured droplet (red curve). For each curve, a picture of the drop encapsulating the maximum volume of oil is shown. (c) Stability diagram for armoured droplets that are: (green region) stable, (blue region) floating or (red region) pinching off. The blue line represents the separatrix between stable and floating regions defined by $\left(\Sigma \mathcal{D} / 4 \pi \ell_{\mathrm{c}}^{2}\right) \leq\left(\mathrm{Bo}^{3 / 2} / 3\right)$, while the red line is the separatrix between stable and pinching off regions defined by $\Sigma \mathcal{D} / 4 \pi \ell_{\mathrm{c}}^{2} \leq\left((2 / 3)\left(\mathrm{Bo}^{3 / 2} / 0.25 * 1.62\right)\right)^{1 / 0.42}$, where $f\left(w / 2 V_{\text {oil }}^{1 / 3}\right)$ is considered constant equal to 0.25 .

tension force, acting along the perimeter of the effective contact line (circumference $\approx \pi w$ ), and pinch-off is expected. The force balance at the contact line can then be expressed as $V_{\text {oil }}\left(\rho_{\mathrm{w}}-\rho_{\mathrm{o}}\right) g=\pi \gamma_{\mathrm{ow}} w \cdot f\left(w / 2 V_{\text {oil }}^{1 / 3}\right)$, where $f\left(w / 2 V_{\text {oil }}^{1 / 3}\right)$ is the capillary correction factor of Harkins and Brown ${ }^{26}$ at detachment. To find a second condition to define a stability diagram for armoured droplets, we measure experimentally $w / \ell_{c}$ which is a function of Bo and $\Sigma \mathcal{D} / 4 \pi \ell_{\mathrm{c}}^{2}$. Just before the droplet breaks up or floats, however, we observe that $w$ is only a function of $\Sigma \mathcal{D} / 4 \pi \ell_{\mathrm{c}}^{2}$, which we fit as $w / \ell_{\mathrm{c}}=1.62\left(\Sigma \mathcal{D} / 4 \pi \ell_{\mathrm{c}}^{2}\right)^{0.42}$ (see inset of Fig. 3a). We take $f \approx 0.25$, and so we predict the separatrix between floating and pinching off as shown in the stability diagram shown in Fig. 3c. We note that recent studies have highlighted the role of gravity on particle stability on droplet interfaces ${ }^{27}$. The authors of that study found that particles at the bottom of a floating droplet are ejected from the interface, because of the weight of the upper layer, when a critical Bond number of 0.01 , defined at the scale of the particles, is reached. Our experiments have focused on smaller particle Bond numbers, hence particle ejection is not observed.

Armoured droplets as a sustainable approach to oil spills. Our observations suggest a sustainable, non-chemical approach to encapsulation and handling of hazardous and volatile liquids that pre-exist as layers in many environmental contexts. For instance, the failure of the oil-drilling rig Deepwater Horizon in April 2010 and the corresponding massive oil spill in the Gulf of Mexico are reminders of our inability to efficiently contain oil slicks ${ }^{28}$. The hydrocarbons spread in thin layers, cover vast areas and reach coastlines where they threaten fragile ecosystems. Current treatment methods include using detergent-like chemicals ${ }^{29}$, direct burning and, potentially, microorganisms ${ }^{30}$. Also, oil skimmers and protective booms seek to contain the oil ${ }^{28}$. All of these approaches have side effects, even when applied successfully. For example, detergents disperse the oil at micron scales, which can be toxic to fish and other animals, burning oil releases toxic fumes, and bioremediation is environmentally sustainable but slow relative to the speed of oil spreading.

We speculate that the destabilization of particle-covered rafts could represent a simple and cheap containment technique to maintain an oil spill geographically localized for its further remediation and to encapsulate the oil into small particle-covered droplets, which either help with eventual remediation or are themselves recoverable. To support this idea, we demonstrate that dry natural sand sprinkled on a centimeter-thick layer of motor oil, representative of environmental cleanup, forms unstable rafts and armoured oil droplets, which sediment and remain stable in sea water (Fig. 4; a detailed calculation of the encapsulated oil volume is given in Methods). In contrast, when we pour a handful of sand or other particles rather rapidly or in clumps into the upper oil layer, the particles are only slowed down by the oilwater interface and are otherwise disorganized, leading to the sedimentation of slurry-like masses (Fig. 4b). The buoyant oil that is not trapped returns to the top of the water column. This failure to encapsulate the oil layer successfully is analogous to the legally prohibited use of oil-sinking agents in the United States, or slurries of chemical, sand and water ${ }^{31}$, which act on the bulk oil layer, but only temporarily sink it. Our approach via the initial destabilization of particle-covered rafts (Fig. 1) underlines the significance of capillary interactions for clean and successful encapsulation and represents a complementary method to other treatments of oil spills using detergent-like chemicals ${ }^{28}$, direct burning, microorganisms $\mathrm{s}^{30}$ and oil skimmers and protective booms $^{28,29}$.

Control of the encapsulation process. Although spontaneous encapsulation could be useful in remediation of hazardous materials, it produces intermittently polydisperse armoured 

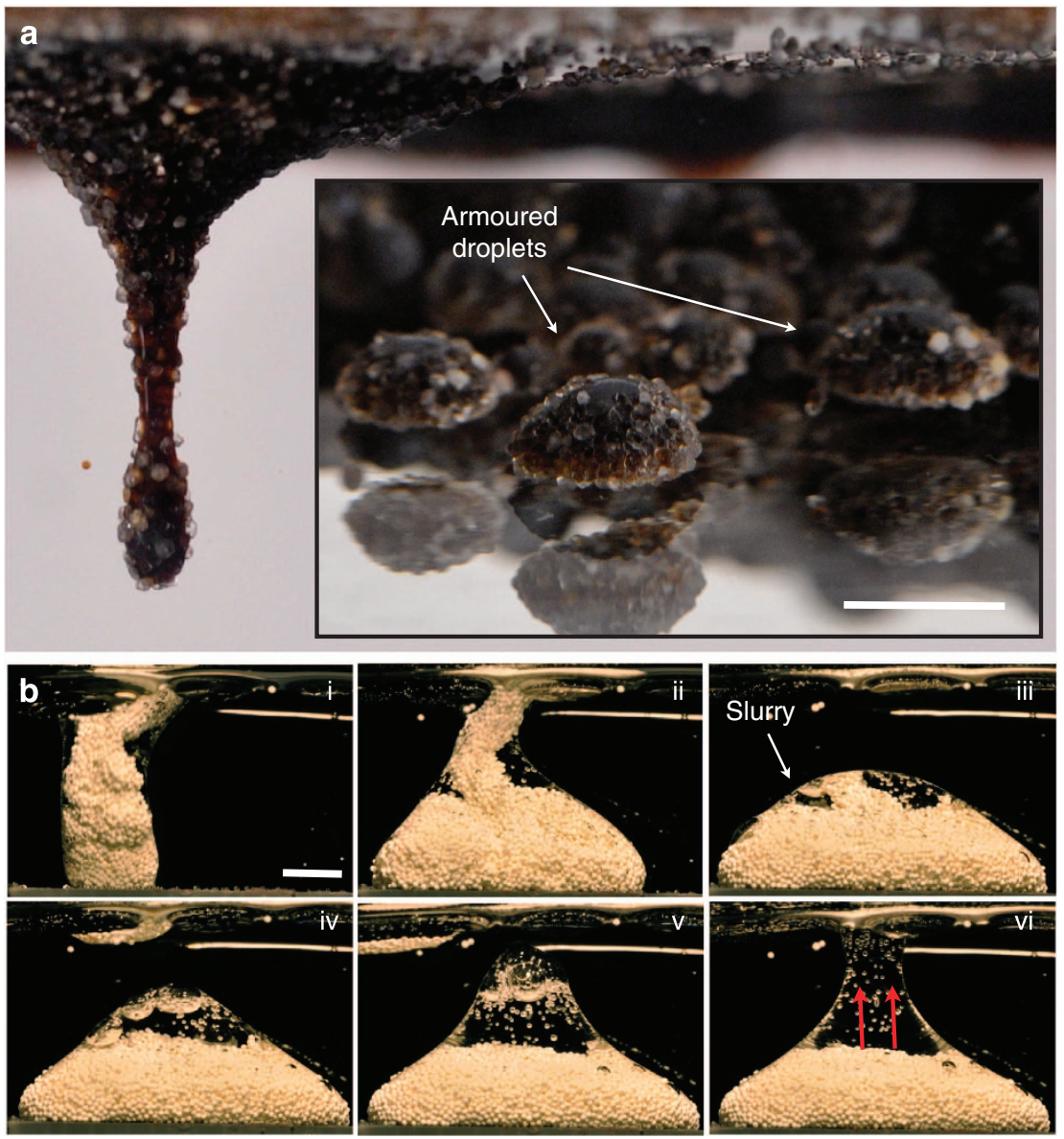

Figure 4 | Slurries versus encapsulation using sand and motor oil. (a) Uniform sprinkling of dry beach sand onto a few millimetre-thick slick of black motor oil floating at the surface of sea water. A capillary raft is formed and destabilizes into armoured droplets that remain stable in the water column. Inset: close-up of armoured droplets after several sinking events. Scale bar, $5 \mathrm{~mm}$ (in $\mathbf{a}$ and $\mathbf{b}$ ). (b) Time sequence of the irregular pouring of zirconia particles $(a=0.175 \pm 0.025 \mathrm{~mm})$ into a layer of oil floating on water. Time lapse of $1 \mathrm{~s}$ between images. Without careful sprinkling of the particles on the oil layer, the oil sinks with the sand (i-iii), forming a slurry, with most of the oil returning to the surface after several seconds (iv-vi).

droplets both in size and surface area covered, and may not be suitable for large scale applications involving encapsulation, transport or controlled chemical reactors. We next demonstrate an approach for continuous encapsulation that does not depend on random disturbances, but is controllable in size, coverage and frequency, and begins with a raft of particles. We deform the raft by pushing down from above with a cylinder of diameter $2 R$ to reach a depth $h_{\text {cyl }}$ (Fig. 5a). At a critical value of the product $h_{\text {cyl }} R$, the raft is destabilized (Fig. 5c and Supplementary Fig. S2). A steady stream of particles of average velocity $v_{\text {part }}$ is generated, which forms armoured droplets continuously at the tip of the cylinder (Fig. 5b and Supplementary Movie 3). $v_{\text {part }}$ does not depend on the velocity at which the cylinder is pushed down on the raft (lower inset Fig. 5c) and it is an increasing function of $h_{\text {cyl }} R$. The surface area of the shell is larger than the surface area of the sphere with the equivalent oil volume so that the droplets fold like deflated balloons, whereas the particles become jam$\mathrm{med}^{18}$, leading to mechanically stable armoured droplets. For a fixed depth $h_{\text {cyl }}$, the smaller the $R$, the more deflated are the armoured droplets (Fig. 5c (i), (ii) and (iii) and Supplementary Fig. S2). When the raft has almost sunk, the tension at the border of the raft changes drastically and we observe that the velocity $v_{\text {part }}$ decreases quickly to zero. To maintain the dripping indefinitely until the upper liquid layer is removed completely, we therefore need to continue sprinkling particles at the border of the raft.
The sedimentation of the particles at the constant speed $v_{\text {part }}$ drags the oil layer downward in a continuous stream. We calculate the tangential viscous stress acting at the oil/water interface, taking into account the buoyancy of the oil as well as the supplementary pressure gradient $\partial_{\mathrm{z}} p: \sigma_{\mathrm{rz}}=\left(\left(\Delta \rho_{\mathrm{wo}} g+\partial_{\mathrm{z}} p\right) \varepsilon\right) / 2$ $+\left(\mu_{\mathrm{o}} v_{\text {part }} / \varepsilon\right)$, where $\varepsilon$ is the thickness of the layer between the sedimenting particle sheath and the static cylinder. To have an estimate of $v_{\text {part }}$, we write a balance between the viscous drag force $\sigma_{\mathrm{rz}} 2 \pi R h_{\text {cyl }}$ and the averaged weight of the sheath that we estimate to be $2 \pi R h_{\text {cyl }} \eta_{\text {cyl }}(4 / 3) \Delta \rho_{\text {pw }} g a$. We take $\eta_{\text {cyl }}=0.5$, the surface fraction of particles along the cylinder. This leads to $\nu_{\text {part }} / \nu_{\text {Stokes }}=6\left(\varepsilon \mu_{\mathrm{w}} / a \mu_{\mathrm{o}}\right) \eta_{\text {cyl }}\left(1-\left(\varepsilon / 2 \mathcal{D}_{\text {cyl }} l_{\mathrm{c}}\right)\left(1+\left(\partial_{\mathrm{z}} p / \Delta \rho_{\text {wo }} g\right)\right)\right)$, where $v_{\text {Stokes }}$ is the Stokes sedimentation velocity of the particles in water defined by $v_{\text {Stokes }}=(2 / 9)\left(\Delta \rho_{\text {pw }} g a^{2} / \mu_{\mathrm{w}}\right)$ and $\mathcal{D}_{\text {cyl }}$ here takes into account the effective thickness of the sheath. If we assume that on the length $h_{\text {cyl }}$, an extra pressure gradient pushes the oil downward due to the variation of the effective surface tension in the raft, which is very low compared with the surface tension of the sheath where it is of the order of $\gamma_{\text {ow }}$, we write that $\partial_{\mathrm{z}} p=\left(\Delta P / h_{\mathrm{cyl}}\right)=\left(\gamma_{\mathrm{wo}} / h_{\mathrm{cyl}} R\right)$. Therefore, we find that

$$
\frac{v_{\text {part }}}{v_{\text {Stokes }}}=6 \frac{\varepsilon \mu_{\mathrm{w}}}{a \mu_{\mathrm{o}}} \eta_{\text {cyl }}\left(1-\frac{\varepsilon}{2 \mathcal{D}_{\mathrm{cyl}} \ell_{\mathrm{c}}}\left(1+\frac{\ell_{\mathrm{c}}^{2}}{R h_{\mathrm{cyl}}}\right)\right)
$$

This estimate, with $\varepsilon=8 a$, fits our data quite well (Fig. 5c). 
a

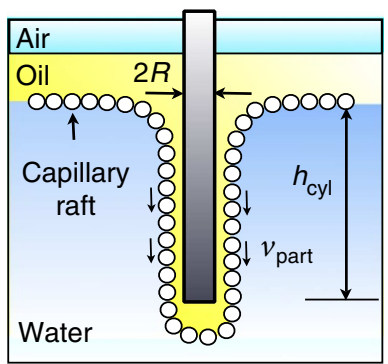

b

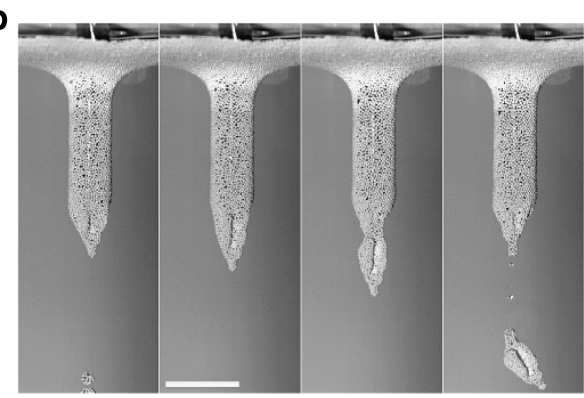

d

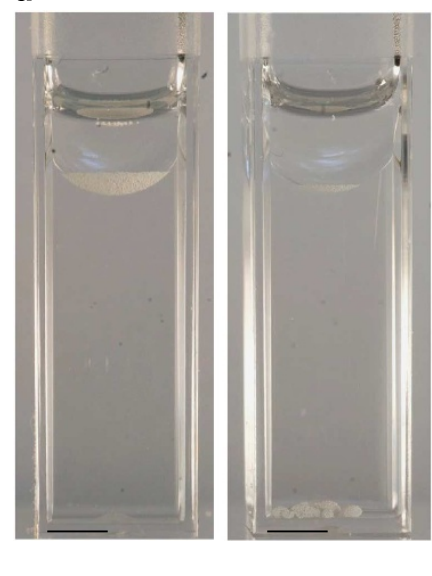

Figure 5 | Controlling capillary encapsulation. (a) Schematic of controlled droplet formation. The raft is deformed by a circular rod (radius $R$ ) to initiate destabilization. (b) Armoured droplets are formed with a uniform size and at a well-defined frequency (mineral oil encapsulated in zirconia particles of radius $a=0.175 \mathrm{~mm}$ ); $R=2.1 \mathrm{~mm}$; time lapse between pictures: $85 \mathrm{~ms}$. Scale bar, $1 \mathrm{~cm}$. (c) The vertical speed of the particles, $v_{\text {part, }}$ non-dimensionalized by $v_{\text {Stokes }}=(2 / 9)\left(\rho_{\mathrm{s}}-\rho_{\mathrm{w}} / \mu_{\mathrm{w}}\right) g a^{2}$, as a function of $h_{\mathrm{cyl}} R / \ell_{\mathrm{c}}^{2}$, following deformation of the raft by rods of increasing radius at a constant depth $h_{\mathrm{cyl}}$ (circles: $h_{\mathrm{cyl}}=19 \mathrm{~mm}$, triangles: $h_{\mathrm{cyl}}=24 \mathrm{~mm}$ and diamonds: $h_{\mathrm{cyl}}=38 \mathrm{~mm}$ ) or constant radius and increasing depths (open symbols, circles: $R=1.207 \mathrm{~mm}$, triangles: $R=1.702 \mathrm{~mm}$ and diamonds: $R=2.286 \mathrm{~mm}$ ). The black curve corresponds to the fit obtained using equation (3). Top inset: armoured droplets formed by dripping from the tip of a rod of decreasing radius (i) $R=2.8 \mathrm{~mm}$, (ii) $R=2.1 \mathrm{~mm}$ and (iii) $R=1.88 \mathrm{~mm}$ at $h_{\text {cyl }}=24 \mathrm{~mm}_{\text {and }} v_{\text {part }}=60 \mathrm{~mm} \mathrm{~s}{ }^{-1}$ have a decreasing surface coverage. All of the results use zirconia particles with radius $a=0.175 \pm 0.025 \mathrm{~mm}$ and $\rho_{\mathrm{s}}=3,800 \mathrm{~kg} \mathrm{~m}^{-3}$. Lower inset: two different destabilizations of the raft to the depth $h_{\text {cyl }}=19 \mathrm{~mm}$ lead to the same constant vertical speed of the particles $v_{\text {part }}=30 \mathrm{mms}^{-1}$ (with $R=1.207 \mathrm{~mm}$, black and red curves) and two different destabilizations at $h_{\text {cyl }}=38 \mathrm{~mm}$ with $R=1.88 \mathrm{~mm}$ lead to the constant velocity $v_{\text {part }}=70 \mathrm{~mm} \mathrm{~s}{ }^{-1}$ (green and curves). (d) The destabilization of a raft of particles with radius $a=0.1 \mathrm{~mm}$ using centrifugal force ( $30 \mathrm{~g}$ ) forms armoured droplets of $1 \mathrm{~mm}$. Scale bar, $0.5 \mathrm{~cm}$.

\section{Discussion}

For particles that are very small and/or are not very dense, particle rafts do not undergo any deformation and cannot be destabilized by gravitational acceleration alone. Both capillary forces and buoyancy acting on the oil prevent the formation of armoured droplets. To enable sinking, looking at the expression for $\mathcal{D}$, one easy way is to change artificially the value of $\ell_{c}$. For example, we placed a raft of zirconia particles of diameter $0.1 \mathrm{~mm}$ in a centrifuge. The raft destabilizes when the acceleration reaches $30 \mathrm{~g}$ and armoured droplets of diameter approximately $1 \mathrm{~mm}$ are formed, which are fives times smaller than $\ell_{c}$ at rest (Fig. 5d). Thus, we have demonstrated that another external force such as an acceleration can induce destabilization. More generally, $\mathcal{D}$ compares the coupled effect of the body force acting at the scale of the particles and the buoyancy and capillary forces counterbalancing it at the scale of the raft. If we replace gravitational force $\left(\rho_{\mathrm{s}}-\rho_{\mathrm{w}}\right) g$ acting on the particles by a general body force $F$, then $\mathcal{D}$ can be expressed as $\left(\ell_{\mathrm{c}} / \ell_{\mathrm{c}}^{*}\right) \mathrm{Bo}_{\mathrm{p}}^{1 / 2}$, where $\ell_{\mathrm{c}}^{* 2}=\gamma / F$ is a new capillary length defined with $F$ and $\mathrm{Bo}_{\mathrm{p}}$ is a particle-scale Bond number defined as $\mathrm{Bo}_{\mathrm{p}}=b / \ell_{\mathrm{c}}^{*}$.

The body-force-driven, capillary encapsulation approach that we have demonstrated provides a means using armoured droplets to handle, transport and isolate liquids originally present as thin floating layers. Our approach has no theoretical limitation of scales and could in principle be extended to the colloidal limit. The ideas are potentially applicable to industrial processes, environmental remediation and sustainable technological practice, especially as only natural materials such as sand are necessary. The impact of other body forces (for example, electric and magnetic) and multiple layers, and strategies for controlled release, offer future directions for research and development.

\section{Methods}

Experimental. A tank of dimensions $6 \times 6 \times 8$ inches is filled with ultrapure water $\left(18.2 \mathrm{M} \Omega \mathrm{cm}\right.$ at $\left.25^{\circ} \mathrm{C}\right)$. A layer of silicone oil (density $\rho=970 \mathrm{~kg} \mathrm{~m}^{-3}$, silicone oil-water interfacial tension $\gamma_{\text {ow }}=30 \mathrm{mN} \mathrm{m}^{-1}$ ) or light mineral oil (density $\rho=838 \mathrm{~kg} \mathrm{~m}^{-3}$, mineral oil-water interfacial tension $\gamma_{\mathrm{ow}}=51 \mathrm{mN} \mathrm{m}^{-1}$ and viscosity $\mu_{\mathrm{o}}=30 \mathrm{mPas}$ ) of thickness $\approx 1 \mathrm{~cm}$ is then added to the top of the water bath. The particles used in these experiments are dense, roughly spherical zirconia beads. They are either made of $69 \%$ zirconia and are slightly polydisperse with $\rho_{\mathrm{s}}=3,800 \mathrm{~kg} \mathrm{~m}^{-3}$ and $a=0.175 \pm 0.025$ or $0.35 \pm 0.05 \mathrm{~mm}$, or are made of $95 \%$ zirconia with $\rho_{\mathrm{s}}=6,000 \mathrm{~kg} \mathrm{~m}^{-3}$ with $a=0.2 \mathrm{~mm}$. Some experiments use sand from the New Jersey shore. Particles are sprinkled far apart from each other to allow the particles to arrive at the interface independently and then interact via capillary forces to form a monolayer of particles at the oil-water interface. To form 
an axisymmetric raft, a small amount of particles is added progressively to the raft along its border.

Calculation of the quantity of sand needed to contain 11 of oil. The formation of particle-covered droplets using the instability of capillary rafts offers a new method of encapsulation, is applicable over large areas and has potential for oil remediation. We can create rafts using non-toxic materials such as sand that allows encapsulation of spilled oil (Fig. 4a). Because the number of particles needed to cover an oil droplet of radius $R_{0}$ is $4\left(R_{0} / a\right)^{2}$, then to cover a centimetre-size droplet with 100 or $500-\mu \mathrm{m}$ diameter particles requires 40,000 or 1,600

particles, respectively. Hence, to contain 11 of oil as armoured droplets requires 0.5 or $0.1 \mathrm{~kg}$ of sand, which makes this idea scalable for major oil spills.

Theoretical. We denote by $h(r)$ the height of the liquid-liquid interface below the top of the drop (see Fig. 2). A normal stress balance gives an expression for the shape of the liquid-liquid interface,

$$
\gamma_{\text {ow }} \nabla \cdot \mathbf{n}=p_{0}-\left(\rho_{\mathrm{w}}-\rho_{\mathrm{o}}\right) g h,
$$

where $p_{0}$ is the pressure inside the droplet at its apex, $g$ is the gravitational acceleration and $\nabla \cdot \mathbf{n}$, with $\mathbf{n}$ the unit normal, is twice the mean curvature of the interface, which can be expressed in terms of $h(r)$ and its derivatives. Along the particle-laden interface, the weight of the particle gives rise to an effective tension that decreases with depth (or alternatively, decreases with distance $s$ along the interface) according to the tangential stress balance

$$
\frac{\mathrm{d} T}{\mathrm{~d} s}=-\left(\rho_{\mathrm{s}}-\rho_{\mathrm{w}}\right) g b_{\mathrm{eff}} \sin \phi,
$$

where $\phi$ is the angle of inclination with respect to the horizontal and $b_{\text {eff }}$ is the weight-averaged effective thickness of the armour, with $\eta$ being the packing fraction. In writing equation (5) and in what follows, we assume for simplicity that the particles displace the outer liquid phase, rather than some combination of the two liquid phases.

As $\sin \phi=\mathrm{d} h / \mathrm{d} s$, we may integrate equation (5) to find an expression for the tension along the armoured region of the droplet,

$$
T(h)=\gamma_{\text {ow }}-\left(\rho_{\mathrm{s}}-\rho_{\mathrm{w}}\right) g b_{e f f}\left[h-h^{*}\right],
$$

where $h^{*}$ is the depth of the upper perimeter of the armoured region (Fig. 2). A balance of normal stresses along the particle-laden interface gives

$$
T(h) \nabla \cdot \mathbf{n}=p_{0}-\left(\rho_{\mathrm{w}}-\rho_{\mathrm{o}}\right) g h-\left(\rho_{\mathrm{s}}-\rho_{\mathrm{w}}\right) g b_{\text {eff }} \cos \phi,
$$

which accounts for the weight of the armour and its variable tension, and reduces to equation (4) in the limit of $T(h)=\gamma$ and $b_{\text {eff }}=0$, corresponding to a droplet with no shell.

The shape of an armoured droplet is therefore given by equation (4) for $0 \leq h \leq h^{*}$, with the initial condition $h(0)=0$ and $h_{\mathrm{r}}(0) \rightarrow 0$, corresponding to a zero position and an infinite slope, respectively (subscripts denote derivatives). Conversely, for $h \geq h^{*}$, the particles occupy the interface, and the shape is given by equation (7) with $T(h)$ determined by equation (6). Finally, at $h=h^{*}$ we require a continuous position, slope and tension.

Next, we non-dimensionalize the governing equations (4), (6) and (7), by letting $H=h / \ell_{\mathfrak{c}}, H^{*}=h^{*} / \ell_{\mathfrak{c}}$, and $R=r / \ell_{\mathfrak{c}}$, where $\ell_{\mathrm{c}}=\sqrt{\gamma_{\mathrm{ow}} /\left(\rho_{\mathrm{w}}-\rho_{\mathrm{o}}\right) g}$, and we define the dimensionless group

$$
\mathcal{D}=\left(\frac{\rho_{\mathrm{s}}-\rho_{\mathrm{w}}}{\rho_{\mathrm{w}}-\rho_{\mathrm{o}}}\right) \frac{b_{\text {eff }}}{\ell_{\mathrm{c}}} .
$$

Doing so yields an expression for the shape of the armour-free region,

$$
\left(\frac{-R_{\mathrm{HH}}+\left(1+R_{\mathrm{H}}^{2}\right) / R}{\left(1+R_{\mathrm{H}}^{2}\right)^{3 / 2}}\right)=P_{0}-H,
$$

and an expression for the shape of the armoured region,

$$
\left(1-\mathcal{D}\left(H-H^{*}\right)\right)\left(\frac{-R_{\mathrm{HH}}+\left(1+R_{\mathrm{H}}^{2}\right) / R}{\left(1+R_{\mathrm{H}}^{2}\right)^{3 / 2}}\right)=P_{0}-H-\frac{\mathcal{D} R_{\mathrm{H}}}{\sqrt{1+R_{\mathrm{H}}^{2}}},
$$

where $P_{0}=p_{0} \ell_{c} / \gamma_{\text {ow }}$ and where we have combined equations (6) and (7). Numerically, it is convenient to work with $R(H)$ rather than $H(R)$ and subscripts denote derivatives. The associated boundary conditions become $H(0)=0$ and $R H(0) \rightarrow \infty$.

The boundary-value problem given by equations (9) and (10) may be solved numerically to determine the shape of an armoured droplet. In addition to $\mathcal{D}$, the solution depends on $P_{0}$ and $H^{*}$, or alternatively, the volume of oil inside the droplet and the surface area of the armoured region. Moreover, we find theoretically that the raft's stability may also be determined by the dimensionless parameter $\mathcal{D}$. When $\mathcal{D} \gtrsim 4$, we find that the raft can only reach a size comparable to the capillary length $\ell_{\mathrm{c}}$ before destabilization occurs (Fig. If and Supplementary Movie 1), and when $\mathcal{D}<4$, the raft destabilizes into an 'interfacial granular jet' (Fig. 1g and
Supplementary Movie 2). We note that for $\mathcal{D} \ll 1$ no spontaneous sinking is observed for any of our experiments.

\section{References}

1. Alargova, R. G., Warhadpande, D. S., Paunov, V. N. \& Velev, O. D. Foam super stabilization by polymer microrods. Langmuir 20, 10371-10374 (2004).

2. Bowden, N., Arias, F., Deng, T. \& Whitesides, G. M. Self-assembly of micro scale objects at a liquid/liquid interface through lateral capillary forces. Langmuir 17, 1757-1765 (2001).

3. Loudet, J.-C. \& Pouligny, B. Self-assembly of capillary arrows. Europhys. Lett. 85, 28003 (2009).

4. Cavallaro Jr., M., Botto, L., Lewandowski, E. P., Wang, M. \& Stebe, K. J. Curvature-driven capillary migration and assembly of rod-like particles. Proc. Natl Acad. Sci. USA 108, 52-20923 (2011).

5. Stamou, D., Duschl, C. \& Johannsmann, D. Long-range attraction between colloidal spheres at the air-water interface: the consequence of an irregular meniscus. Phys. Rev. E 62, 625263 (2000).

6. Kralchevsky, P. A., Denkov, N. D. \& Danov, K. D. Particles with an undulated contact line at a fluid interface: interaction between capillary quadrupoles and rheology of particulate monolayers. Langmuir 17, 7694-7705 (2001).

7. Danov, K. D., Kralchevsky, P. A. \& Boneva, M. P. Electrodipping force acting on solid particles at a fluid interface. Langmuir 20, 6139-6151 (2004).

8. Nikolaides, M. G. et al. Electric-field induced capillary attraction between likecharged particles at liquid interfaces. Nature 420, 299-301 (2002).

9. Zahn, K., Mendez-Alcaraz, J. M. \& Maret, G. Hydrodynamic interactions may enhance the self-diffusion of colloidal particles. Phys. Rev. Lett. 79, 175-178 (1997).

10. Kralchevsky, P. A. \& Nagayama, K. Capillary interactions between particles bound to interfaces, liquid films and biomembranes. Adv. Colloid Interface Sci. 85, 145-192 (2000).

11. Mansfield, E. H., Sepangi, H. R. \& Eastwood, E. A. Equilibrium and mutual attraction or repulsion of objects supported by surface tension. Phil. Trans. $R$ Soc. Lond. A 355, 868-919 (1997).

12. Planchette, C., Lorenceau, E. \& Biance, A.-L. Surface wave on particle raft. Soft Matter 8, 2444-2451 (2012).

13. Vella, D., Metcalfe, P. D. \& Whittaker, R. J. Equilibrium conditions for the floating of multiple interfacial objects. J. Fluid Mech. 549, 215-224 (2006).

14. Aussillous, P. \& Quéré, D. Properties of liquid marbles. Proc. Roy. Soc. A 462, 973-999 (2006).

15. Subramaniam, A. B., Abkarian, M., Mahadevan, L. \& Stone, H. A. Non spherical bubbles. Nature 438, 905-905 (2005).

16. Stratford, K., Adhikari, R., Pagonabarraga, I., Desplat, J.-C. \& Cates, M. E. Colloidal jamming at interfaces: a route to fluid-bicontinuous gels. Science 309, 2198-2201 (2005).

17. Arditty, S., Schmitt, V., Giermanska-Kahn, J. \& Leal-Calderon, F. Materials based on solid-stabilized emulsions. J. Colloid Interface Sci. 275, 659-664 (2004).

18. Subramaniam, A. B., Abkarian, M. \& Stone, H. A. Controlled assembly of jammed colloidal shells on fluid droplets. Nat. Mater. 4, 553-556 (2005).

19. Dorvee, J. R., Derfus, A. M., Bhatia, S. N. \& Sailor, M. J. Manipulation of liquid droplets using amphiphilic, magnetic one-dimensional photonic crystal chaperones. Nat. Mater. 3, 896-899 (2004).

20. Zhao, Y., Fang, J., Wang, H., Wang, X. \& Lin, T. Magnetic liquid marbles: manipulation of liquid droplets using highly hydrophobic $\mathrm{Fe}_{3} \mathrm{O}_{4}$ nanoparticles. Adv. Mater. 22, 707-710 (2010).

21. Aussillous, P. \& Quéré, D. Liquid marbles. Nature 411, 924-927 (2001).

22. Binks, B. P. \& Murakami, R. Phase inversion of particle-stabilized materials from foams to dry water. Nat. Mater. 5, 865-869 (2006).

23. Tcholakova, S., Denkov, N. D. \& Lips, A. Comparison of solid particles, globular proteins and surfactants as emulsifiers. Phys. Chem. Chem. Phys. 10, 1608-1627 (2008)

24. Caballero, M., Cela, R. \& Perezbustamante, J. A. Analytical applications of some flotation techniques - a review. Talanta 37, 275-300 (1990).

25. Singh, P. \& Joseph, D. D. Fluid dynamics of floating particles. J. Fluid Mech. 530, 31-80 (2005).

26. Harkins, W. D. \& Brown, F. E. The determination of surface tension (free surface energy), and the weight of falling drops - the surface tension of water and benzene by the capillary height method. J. Am. Chem. Soc. 41, 499-524 (1919).

27. Tavacoli, J. W., Katgert, G., Kim, E. G., Cates, M. E. \& Clegg, P. S. Size limit for particle-stabilized emulsion droplets under gravity. Phys. Rev. Lett. 108, 268306 (2012).

28. Kerr, R., Kintisch, E. \& Stokstad, E. Gulf oil spill: will deepwater horizon set a new standard for catastrophe? Science 328, 674-675 (2010).

29. Schrope, M. Oil spill: deep wounds. Nature 472, 152-1547 (2011).

30. Bragg, J. R., Rince, R. C., Harner, E. J. \& Atlas, R. M. Effectiveness of bioremediation for the Exxon-Valdez oil-spill. Nature 368, 413-418 (1994).

31. Blackman, R. A. A. Toxicity of oil-sinking agents. Mar. Pollut. Bull. 5, 116-118 (1974). 


\section{Acknowledgements}

We thank M. Roché for his assistance with the experiments and W. H. Gowen for background on oil-sinking agents. J.M.A. acknowledges funding from the NSF Mathematical Sciences Postdoctoral Research Fellowship Program. We thank Princeton University, the Princeton Environmental Institute and CNRS for support of this research. H.A.S. thank the NSF CBET- 1234500 for partial support of this work.

\section{Author contributions}

M.A., S.P., J.M.A. and H.A.S. designed the concepts. M.A. and S.P. carried out the experiments. M.A., S.P., J.M.A. and H.A.S. developed the models. M.A., S.P., J.M.A. and H.A.S. discussed and interpreted results. M.A., S.P., J.M.A. and H.A.S. wrote the manuscript.

\section{Additional information}

Supplementary Information accompanies this paper at http://www.nature.com/ naturecommunications

Competing financial interests: The authors declare no competing financial interests.

Reprints and permission information is available online at http://npg.nature.com/ reprintsandpermissions/

How to cite this article: Abkarian, M. et al. Gravity-induced encapsulation of liquids by destablization of granular rafts. Nat. Commun. 4:1895 doi: 10.1038/ncomms2869 (2013). 